\title{
Mean stress sensitivity of ductile iron with respect to technological and statistical size effect considering defects
}

\author{
Paul Kainzinger ${ }^{\mathrm{a}}$ and Florian Grün \\ Chair of Mechanical Engineering, Montanuniversität Leoben, 8700 Leoben, Austria
}

\begin{abstract}
Specimens of two sizes have been taken from two sampling locations within a wind turbine hub made of nodular cast iron (EN-GJS-400-18-LT) for constant amplitude fatigue testing. The sampling positions exhibit varying cooling conditions, resulting in different microstructures. Fatigue tests have been carried out at $\mathrm{R}$-ratios of $\mathrm{R}=-1$ and $\mathrm{R}=0$. The coarse microstructure as well as the larger specimens yielded in lower fatigue strengths. No effect of the microstructure or the specimen size on the mean stress sensitivity has been found. Fractographic analysis of the fractured specimen's surface revealed microshrinkages to be the source of crack initiation for all specimens. Micro-shrinkage size increases from fine to coarse microstructure and with increasing specimen size. The ElHaddad equation using the $\sqrt{ }$ area parameter was used to describe the fatigue limit. The results were in good agreement with the experiments.
\end{abstract}

\section{Introduction}

Ductile Iron is widely used throughout different industrial fields due to its beneficial castability, price-performance ratio and availability. Components made of ductile Iron are often subjected to dynamic loads. Superimposed mean stresses significantly influence the durable stress amplitude. The characterization of the mean stress sensitivity of the ductile iron EN-GJS-400-18-LT is subject of the present paper.

\section{Experimental procedure}

Two differently sized round bar specimen have been taken from a wind turbine hub made of EN-GJS400-18-LT. The volume stressed with $90 \%$ of the peak stress [1] is $950 \mathrm{~mm}^{3}$ for the small specimen and $5.000 \mathrm{~mm}^{3}$ for the large specimen. The fatigue tests have been carried out under tension-compression loading until $10^{7}$ load cycles using $\mathrm{R}$-ratios of $\mathrm{R}=-1$ and $\mathrm{R}=0$. Specimens have been taken from two different microstructures. The first is a coarse microstructure with few, large nodules per square millimetre, the second represents a fine microstructure with many but small nodules per square millimetre. Both microstructures have a fully ferritic matrix. A detailed description of the experimental procedure, specimen sampling location, as well as the evaluation of the fatigue limit can be found in [2].

\footnotetext{
${ }^{a}$ Corresponding author: paul. kainzinger@unileoben.ac . at
}

This is an Open Access article distributed under the terms of the Creative Commons Attribution License 4.0, which permits unrestricted use, distribution, and reproduction in any medium, provided the original work is properly cited. 

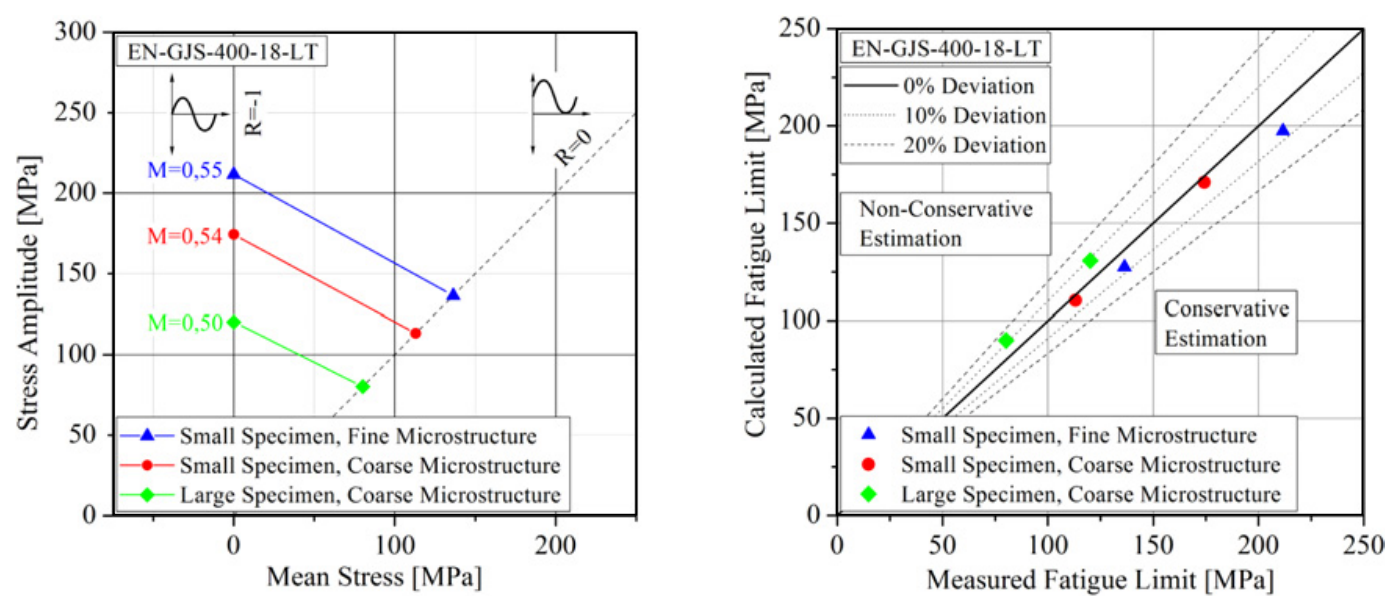

Figure 1. Haigh-diagram of the fatigue tests (left). Relation of the measured and calculated fatigue limits (right).

\section{Results}

The results of the fatigue tests are shown in Fig. 1 (left). Larger specimens, as well as a coarse microstructure result in a lower fatigue strength. The mean stress sensitivity calculated according to [3] shows no dependency on neither the microstructure, nor the specimen size. It shall be pointed out, that common guidelines and codes $[4,5]$ recommend significantly lower mean stress sensitivities of $\mathrm{M}=0,2$ which lead to non-conservative results.

\section{Discussion}

Micro-shrinkages have been identified as the source of crack initiation in the fractured specimen's surface for all specimens using a SEM. The size of these micro-shrinkages has been quantified using the $\sqrt{ }$ area parameter [6]. This size increases with larger specimens and coarser microstructure. The ElHaddad equation [7] has been used to estimate the fatigue limit based on supplementary crack growth experiments [2]. The calculated fatigue limits are in good agreement with the measured fatigue limits (cf. Fig. 1, right).

\section{References}

[1] C. M. Sonsino, Konstruktion 45, p22-33 (1993)

[2] P. Kainzinger, Ph.D.-thesis, Leoben (2013)

[3] W. Schütz, Zeitschrift für Flugwissenschaften 15 p407-419 (1967)

[4] Forschungskuratorium Maschinenbau e.V, Frankfurt am Main 6 (2012)

[5] Germanischer Lloyd, Guideline for the Certification of Wind Turbines (2003)

[6] Y. Murakami, Int. J. Fatigue 41 p2-10 (2012)

[7] M. El Haddad, K. Smith, T. Topper, ASME transactions 101 (1979) 